\title{
Optimal Control of a Discrete HIV Model with Two Classes of Antiretroviral Therapy
}

\author{
Amine Bouaine ${ }^{1}$, Mostafa Rachik ${ }^{2}$ \\ ${ }^{2}$ Laboratory of Analysis Modeling and Simulation, Department of Mathematics and Computer Sciences, Faculty of Sciences Ben M'Sik, \\ Hassan II Mohammedia University BP 7955, Sidi Othman, Casablanca, Morocco
}

\begin{abstract}
The aim of this work is to set an adequate discrete HIV models taking into consideration two antiretroviral: Protease Inhibitors and Reverse Transcriptase Inhibitors. Furthermore, we investigate optimal control strategies in order to reduce infected cells and infectious virions. Using an iterative method, three numerical simulations are carried out, compared and interpreted to confirm the the proposed model and to prove the performance of the optimization strategies. Finally, we improve the patients' lives by minimizing serious side effects and also reducing drug costs and duration of treatments.
\end{abstract}

Keywords: Optimal control, Therapy, Discrete HIV model, Pontryagin's Maximum Principle

\section{Introduction}

HIV (human immunodeficiency virus) is the virus that causes AIDS (acquired immunodeficiency syndrome). HIV kills and damages cells of the body's immune system and destroys progressively the body's ability to fight infections and certain cancers. AIDS appeared for the first times in 8081 years in the United States. A few years later (in 1983), HIV was highlighted at the Pasteur Institute in Paris and recognized as responsible for this syndrome. Today, over 36 million people living with HIV in the world. The latest statistics of 2014 are detailed in Table 1, according to the World Health Organization (WHO).

Table 1: Global summary of the AIDS epidemic 2014

\begin{tabular}{|l|c|c|c|}
\hline & Adults & children & Total \\
\hline $\begin{array}{l}\text { Number of people (million) living } \\
\text { with HIV in 2014 }\end{array}$ & 34.3 & 2.6 & 36.9 \\
\hline $\begin{array}{l}\text { People newly infected (million) } \\
\text { with HIV in 2014 }\end{array}$ & 1.8 & 0.2 & 2.0 \\
\hline AIDS deaths (million) in 2014 & 1.0 & 0.15 & 1.2 \\
\hline
\end{tabular}

The immune system is a complex set of organs, cells and molecules. It is responsible for the body's defenses against infectious agents. The main components of the immune system to which we will interest are $\mathrm{CD}_{4}^{+} \mathrm{T}$ lymphocytes. We are interested in mathematical models considering the dynamic contribution of these cells.

CD4 T-cells (or T4), also known as helper T cells (Th) or Helper $\mathrm{T}$, are the cells responsible for the coordination and activation of the immune response. These cells are also produced by the thymus and have $\mathrm{CD} 4$ protein on their membranes. These helper cells, as their name suggests, are the key to the immune system. They help to trigger the reaction and the immune response in case of infection.

The different classes of ART (antiretroviral) used against HIV act at different stages of the virus life cycle. The most common antiretroviral are mainly:

- Reverse Transcriptase Inhibitors (RTIs);

- Protease Inhibitors (PIs).
Table 2: Number of people receiving ART and percentage of all people living with HIV receiving ART in low and middle income countries, 2013

\begin{tabular}{|l|c|c|}
\hline Region & $\begin{array}{c}\text { Number of } \\
\text { people } \\
\text { receiving } \\
\text { ART }\end{array}$ & $\begin{array}{c}\text { Percentage } \\
\text { of people } \\
\text { receiving } \\
\text { ART in each } \\
\text { region }\end{array}$ \\
\hline Africa region & $9,100,000$ & 37 \\
\hline South and middle of The Americas & 790,000 & 44 \\
\hline South-East Asia region & $1,100,000$ & 33 \\
\hline Eastern European region & 255,000 & 22 \\
\hline Eastern Mediterranean region & 790,000 & 44 \\
\hline Western Pacific region & 400,000 & 32 \\
\hline
\end{tabular}

Unfortunately, much of infected does not receive ART, especially in countries whose income is low or limited. The Table 2, according to the World Health Organization (WHO), shows clearly this reality.

Mathematical modeling of biological processes aims to better understand complex or often misunderstood phenomena of these bio-processes. A mathematical model is a set of mathematical equations that links; on one side, a set of variables which are states of the system studied for example body temperature, viral load, on the other hand, a set of parameters that are constants or variables specific to the system, for example the mass body, the life of the virus. In addition, the mathematical model takes also into consideration a set of constraints.

In this study, we adopt discrete-time models for many reasons: the statistics data concerning HIV infection are collected in discrete-time, the discrete-time models may escape some mathematical complexities like regularity of solutions, adequate topology and the choice of state space. On top of that, to simulate continuous models, we are in need to discretize the concerned model, this passage (discretization) may not reflect exactly the continuous model. So the discrete-time are more suitable to describe HIV infection than the continuous ones.

Two controls which measure the efficiency of reverse transcriptase inhibitors and protease inhibitors are introduced so new discrete epidemic model with two controls: $u_{1}$ and $u_{2}$, is developed and validated. Furthermore, Pontryagin's 


\section{International Journal of Science and Research (IJSR) \\ ISSN (Online): 2319-7064}

Index Copernicus Value (2013): 6.14 | Impact Factor (2014): 5.611

maximum principle is applied to this discrete HIV model in order to minimize the objective functional defined in (6) by decreasing the number of infected cells, the number of infectious virus and reducing the costs of treatment. Optimal control problem is established to predict efficient economic strategy and prescribe adequate dose of treatment.

In the literature, W. Garira et al. [1] presented continuous model with two controls and the results show that for drugs with less toxicity, continuous therapy is beneficial. H. Joshi [2] presented HIV system of ordinary differential equation but he took into account just two variable states uninfected $\mathrm{CD}_{4}^{+} \mathrm{T}$ cells and free infectious virions. D. Kirschner [3] introduced chemotherapy in an early treatment setting and used a single control, the results don't depend on the treatment duration.

This paper is organized as follows. In the next section, we develop several HIV models until getting the discrete HIV models with two classes of antiretroviral (RTIs) and (PIs). In section 3, the analysis of optimization problem is presented. In section 4, we suggest a numerical appropriate method and the corresponding simulation results. Finally, conclusions are summarized in the last section.

\section{Presentation of Discrete HIV Model with Two Controls}

In this section, different HIV models will be described, explained, discussed and presented. In fact, we show different steps traversed in order to reach the final model this our subject matter.

\subsection{Discrete HIV Model}

In this subsection, we present a discrete HIV model under therapy. The model takes into consideration three variable states described below.

$x_{k}$ uninfected $\mathrm{CD} 4^{+} \mathrm{T}$-cells at time $k$;

$y_{k}$ infected $\mathrm{CD} 4^{+}$T-cells cells at time $k$;

$v_{I_{k} k}$ infectious virus at time $k$.

Uninfected $\mathrm{CD}^{+}{ }^{+}$T-cells $x_{k}$ are produced at a rate $s$ and cleared at a rate $d$. Infectious virus $v_{I_{2} k}$ die at a rate $\mu$ and infected $\mathrm{CD} 4^{+}$T-cells $y_{k}$ die at a rate $a$. The movement from class of uninfected cells to class of infected cells is described by $\beta v_{I_{k} k} x_{k}$, where $\beta$ is the effective contact rate. Hence, the $\beta v_{I_{k} k} x_{k}$ term is subtracted from uninfected CD4 ${ }^{+}$T-cells $x_{k+1}$ and added to infected cells $y_{k+1}$. The virus is produced by infected cells at a rate $K$. Furthermore, through therapy a part of infected cells may also revert to the uninfected state by loss of all cccDNA from their nucleus at a rate $r$. Hence, the model is described as follows

$$
\begin{aligned}
& x_{k+1}=s+x_{k}-\beta v_{I_{l} k} x_{k}-d x_{k}+r y_{k} \\
& y_{k+1}=y_{k}+\beta v_{l_{2} k} x_{k}-(a+r) y_{k} \\
& v_{I_{l} k+1}=v_{l_{l} k}+K y_{k}-\mu v_{l_{l} k}
\end{aligned}
$$

\subsection{Discrete HIV model with Protease Inhibitors (PIs)}

Protease Inhibitors (PIs) are kind of medications used to treat or prevent infection by viruses, in other words, they work by blocking the activity of reverse transcriptase and prevent production of viruses from the actively infected CD4 ${ }^{+}$T-cells, see table 3 .

Table 3: Examples of most common PIs

\begin{tabular}{|c|c|c|}
\hline Abbreviation & International noun & Commercial noun \\
\hline APV & Amprenavir & Agenerase \\
\hline TPV & Indinavir & Crixivan \\
\hline RTV & Ritonavir & Norvir \\
\hline ATZ & Atazanvir & Reyataz \\
\hline
\end{tabular}

Protease Inhibitors (PIs) force part of infected CD4 ${ }^{+} \mathrm{T}$-cells to be converted to noninfectious virions. So, another variable state is added.

$v_{N_{k} k}$ noninfectious virus at time $\mathrm{k}$.

Noninfectious virus die also at a rate $\mu$, and part of infectious virus $\rho K y_{K}$ move to noninfectious virus class where the parameter $\rho$ measures the effectiveness of Protease Inhibitors (PIs). Then, we obtain the following model

$$
\begin{aligned}
& x_{k+1}=s+x_{k}-\beta v_{I_{l} k} x_{k}-d x_{k}+r y_{k} \\
& y_{k+1}=y_{k}+\beta v_{l_{\imath} k} x_{k}-(a+r) y_{k} \\
& v_{l_{\imath} k+1}=v_{l_{l} k}+(1-\rho) K y_{k}-\mu v_{l_{l} k} \\
& v_{N_{\imath} k+1}=v_{N_{\imath} k}+\rho K y_{k}-\mu v_{N_{\imath} k}
\end{aligned}
$$

\subsection{Discrete HIV model with Reverse Transcriptase Inhibitors (RTIs)}

We introduce Reverse Transcriptase Inhibitors (RTIs) in order to prevent infection of new cells. They are also a class of antiretroviral drug used to treat HIV infection. Another new parameter will be included: $\sigma$ with the aim of measuring the effectiveness of Reverse Transcriptase Inhibitors (RTIs), see table 4 .

Table 4: Examples of most common RTIs

\begin{tabular}{|c|c|c|}
\hline Abbreviation & International noun & Commercial noun \\
\hline AZT & Zidovudine & Rtrovir \\
\hline $\mathrm{ddI}$ & Didanosine & Videx \\
\hline $\mathrm{ddC}$ & Zalcitabine & Hivid \\
\hline $\mathrm{d} 4 \mathrm{~T}$ & Staduvine & Zerit \\
\hline
\end{tabular}

In this case, under Reverse Transcriptase Inhibitors (RTIs), the model (1) becomes

$$
\begin{aligned}
& x_{k+1}=s+x_{k}-(1-\sigma) \beta v_{I_{\imath} k} x_{k}-d x_{k}+r y_{k} \\
& y_{k+1}=y_{k}+(1-\sigma) \beta v_{I_{l} k} x_{k}-(a+r) y_{k} \\
& v_{I_{l} k+1}=v_{I_{l} k}+K y_{k}-\mu v_{l_{l} k}
\end{aligned}
$$

\section{Volume 5 Issue 2, February 2016 www.ijsr.net}




\section{International Journal of Science and Research (IJSR) \\ ISSN (Online): 2319-7064}

Index Copernicus Value (2013): 6.14 | Impact Factor (2014): 5.611

2.4 Discrete HIV model with two controls (PIs) and (RTIs)

In this subsection, we introduce two drugs Protease Inhibitors (PIs) and Reverse Transcriptase Inhibitors (RTIs). In fact, the parameters $\sigma$ and $\rho$ depend on time, are respectively considered as control functions $u_{1_{q} k}$ and $u_{2, k}$. $U_{a d}$ is the set of admissible controls given by (4)

$U_{a d}=\left\{u=\left(u_{1}, u_{2}\right) ; 0 \leq u_{i, k} \leq u_{i}^{\max } \leq 1\right\}$

For $k=0, \ldots, T-1$ and $i=1,2$.

As a result, our next model is described as follows

$$
\begin{aligned}
& x_{k+1}=s+x_{k}-\left(1-u_{1_{a} k}\right) \beta v_{I_{a} k} x_{k}-d x_{k}+r y_{k} \\
& y_{k+1}=y_{k}+\left(1-u_{1_{\imath} k}\right) \beta v_{I_{k} k} x_{k}-(a+r) y_{k} \\
& v_{I_{2} k+1}=v_{I_{\imath} k}+\left(1-u_{2, k}\right) K y_{k}-\mu v_{I_{k} k} \\
& v_{N_{k} k+1}=v_{N_{\imath} k}+u_{2, k} \rho K y_{k}-\mu v_{N_{k} k}
\end{aligned}
$$

For biological reasons, we have $x_{0} \geq 0, y_{0} \geq 0, v_{I_{0} 0} \geq 0$, $v_{N, 0} \geq 0$ and $u_{1,0}=u_{2,0}=0$.

Note that $k=0,1, \ldots, T-1$, is the index for the time steps and there are two controls defined as follows $u_{i}=\left(u_{i, 0}, u_{i, 1}, \ldots, u_{i, T-1}\right)$ with $i=1,2$.

\section{The Optimal Control Problem}

In this section, our target is to minimize the objective functional defined in (6) by decreasing the number of infected cells and the number of infectious virus. On top of that, we are interested also in reducing costs of treatment by Protease Inhibitors (PIs) and Reverse Transcriptase Inhibitors (RTIs). We first define $I\left(u_{1}, u_{2}\right)$ as the objective functional given by

$I\left(u=\left(u_{1}, u_{2}\right)\right)=A y_{T}+C v_{I_{2} T}$

$+\sum_{k=0}^{T-1}\left(A y_{k}+C v_{I_{k} k}+\frac{1}{2} B_{1} u_{1, k}^{2}+\frac{1}{2} B_{2} u_{2, k}^{2}\right)$

For simplicity the cost of each treatment is considered quadratic, where $\mathrm{A}, \mathrm{C}, \mathrm{B}_{1}$, and $\mathrm{B}_{2}$ are the cost coefficients and depend respectively on the relative importance of $y_{k}$,

$v_{I_{2} k}, u_{1_{\varepsilon} k}$, and $u_{2, k}$.

The positive constants $\mathrm{A}, \mathrm{C}, \mathrm{B}_{1}$, and $\mathrm{B}_{2}$ represent desired weight on the benefit and cost, $u_{1_{\imath} k}$ and $u_{2, k}$ are bounded, lebesgue integrals functions and reflect the severity of the side effects of the drugs [2]. We are looking for optimal control $u^{*}=\left(u_{1}^{*}, u_{2}^{*}\right)$ which verifies (7)

$$
I\left(u_{1}^{*}, u_{2}^{*}\right)=\min _{\left(u_{1}, u_{2}\right) \in U_{a d}} I(u)
$$

\subsection{Existence of an optimal control}

The existence of the optimal control pair can be obtained using a result by Fleming and Rishel [4] and by Lukes [5].

Theorem 3.1

There exists control function $u^{*}=\left(u_{1}^{*}, u_{2}^{*}\right)$ which verifies

$$
I\left(u_{1}^{*}, u_{2}^{*}\right)=\min _{\left(u_{1}, u_{2}\right)} \in U_{a d} J(u)
$$

\section{Proof}

To prove the existence of an optimal control pair it is sufficient to verify that

1) The set of controls and corresponding state variables is nonempty.

2) The admissible set $U_{a d}$ is convex and closed.

3) The right hand side of the state system (5) is bounded by a linear function in the state and control variables.

4) The integrand of the objective functional is concave on $U_{a d}$.

5) There exists constants $c_{1}>0, c_{2}>0$ and $\rho>1$ such that the integrand $L\left(y, v, u_{1}, u_{2}\right)$ of the objective functional satisfies

$L\left(y, v, u_{1}, u_{2}\right)>c_{2}+c_{1}\left(\left|u_{1}\right|^{2}+\left|u_{2}\right|^{2}\right)^{\rho / 2}$

The result follows directly from (Fleming and Rishel 1975).

In order to verify these conditions, we use a result by Lukes [5] to give the existence of solutions of (5) with bounded coefficients, which gives condition 1 . We note that the solutions are bounded. Our control set satisfies condition 2 . Since our state system is bilinear in $u_{1}$ and $u_{2}$, the model (5) satisfies condition 3 , using the boundedness of the solutions. Note that the integrand of our objective functional is concave. Also we have the last condition needed where $c_{2}$ depends on the upper bound on $y$ and $v$, furthermore $c_{1}>0$ since $B_{1}, B_{2}>0$. We conclude there exists an optimal control pair.

\subsection{Characterization of the Optimal Control}

According to the Pontryagin's Maximum Principal in discrete-time, Equations (5), (6) and (7) will be converted into a problem of minimizing a Hamiltonian $H_{k}$.

At each time step $k$, the Hamiltonian is given by

$$
\begin{gathered}
H_{k}=A y_{k}+C v_{I_{2} k}+\frac{1}{2} B_{1} u_{1_{k} k}^{2}+\frac{1}{2} B_{2} u_{2, k}^{2} \\
+\sum_{j=1}^{4} \lambda_{j_{k} k+1} g_{j, k}
\end{gathered}
$$

Where $g_{j, k}$ is the right side of the difference equation of the $j^{\text {th }}$ state variable at time step $k+1$.

\section{Theorem 3.2}

Given an optimal control $u_{k}^{*}=\left(u_{1_{k} k}^{*}, u_{2, k}^{*}\right) \in U_{a d}$ and solutions $x_{k,}^{*} y_{k}^{*}, v_{l k}^{8}$, and $v_{N k}^{*}$ of the corresponding states system (5), there exist adjoint functions $\lambda_{1_{2} k}, \lambda_{2, k}, \lambda_{3, k}$, and $\lambda_{4, k}$ satisfying

$\lambda_{1, k}=\lambda_{1, k+1}\left[-(d-1)-\left(1-u_{1, k}\right) \beta v_{I_{\imath} k}\right]$

\section{Volume 5 Issue 2, February 2016 www.ijsr.net}




\section{International Journal of Science and Research (IJSR) \\ ISSN (Online): 2319-7064}

Index Copernicus Value (2013): 6.14 | Impact Factor (2014): 5.611

$$
\begin{aligned}
& +\lambda_{2, k+1}\left[\left(1-u_{1, k}\right) \beta v_{l, k}\right] \\
\lambda_{2, k}= & A+\lambda_{1, k+1} r+\lambda_{2, k+1}[(-a-r-1)] \\
& +\lambda_{3, k+1}\left[\left(1-u_{2, k}\right) K\right]+\lambda_{4, k+1}\left[u_{2, k} K\right] \\
\lambda_{3, k}= & C+\left(\lambda_{1, k+1}+\lambda_{2, k+1}\right)\left[-\left(1-u_{1, k}\right) \beta x_{k}\right] \\
& +\lambda_{3, k+1}[-(\mu-1)] \\
\lambda_{4, k}= & \lambda_{4, k+1}[-(\mu-1)]
\end{aligned}
$$

with the transversality conditions $\lambda_{1, T}=\lambda_{4, T}=0$,

$\lambda_{3, T}=C$ and $\lambda_{2, T}=A$.

Furthemore, for $k=0, . ., T-1$ the optimal control

$u^{*}=\left(u_{1}^{*}, u_{2}^{*}\right)$ is given by $(10)$

$u_{1, k}^{*}=\min \left[u_{1}^{\max }, \max \left(0, \beta v_{I_{\ell} k} x_{k}\left(\lambda_{2, k+1}-\lambda_{1, k+1}\right) / B_{1}\right)\right]$

$u_{2, k}^{*}=\min \left[u_{2}^{\max }{ }_{,} \max \left(0, K y_{k}\left(\lambda_{3_{k} k+1}-\lambda_{1, k+1}\right) / B_{2}\right)\right]$

Proof.

The Hamiltonian is given by

$$
\begin{aligned}
& H_{k}=A y_{k}+C v_{I_{k} k}+\frac{1}{2} B_{1} u_{1, k}^{2}+\frac{1}{2} B_{2} u_{2, k}^{2} \\
& +\lambda_{1, k+1}\left(s+x_{k}-\left(1-u_{1, k}\right) \beta v_{l_{l} k} x_{k}-d x_{k}+r y_{k}\right) \\
& +\lambda_{2, k+1}\left(y_{k}+\left(1-u_{1, k}\right) \beta v_{I_{k} k} x_{k}-(a+r) y_{k}\right) \\
& +\lambda_{3, k+1}\left(v_{l_{l, k}}+\left(1-u_{2, k}\right) K y_{k}-\mu v_{l_{l} k}\right) \\
& +\lambda_{4, k+1}\left(v_{N_{g} k}+u_{2, k} \rho K y_{k}-\mu v_{N_{\imath} k}\right)
\end{aligned}
$$

According to the Pontryagin's Maximum Principle in discrete time, see [6], the equations for the adjoint variables, for $k=0,1 . ., T-1$, are

$$
\begin{array}{ll}
\lambda_{1, k}=\frac{\partial H_{k}}{\partial x_{k}}, & \lambda_{1, T}=0 \\
\lambda_{2, k}=\frac{\partial H_{k}}{\partial y_{k}}, & \lambda_{2, T}=A \\
\lambda_{3, k}=\frac{\partial H_{k}}{\partial v_{l, k}}, & \lambda_{3, T}=C \\
\lambda_{4, k}=\frac{\partial H_{k}}{\partial v_{N_{\imath} k}}, & \lambda_{4, T}=0
\end{array}
$$

For $k=0,1 . ., T-1$, the control characterization is derived from (13)

$$
\frac{\partial H_{k}}{\partial u_{1, k}}=B_{1} u_{1, k}+\lambda_{1, k+1}\left(\beta v_{l_{l} k} x_{k}\right)+\lambda_{2, k+1}\left(-\beta v_{l_{l} k} x_{k}\right)
$$

$$
\frac{\partial H_{k}}{\partial u_{2, k}}=B_{2} u_{2, k}+\lambda_{3, k+1}\left(-K y_{k}\right)+\lambda_{4, k+1}\left(K y_{k}\right)=0
$$

Subject to the lower and upper bounds for $u=\left(u_{1}, u_{2}\right)$, the characterization becomes

$$
\begin{aligned}
& u_{1, k}^{*}=\min \left[u_{1}^{\max }, \max \left(0, \beta v_{l_{k} k} x_{k}\left(\lambda_{2, k+1}-\lambda_{1, k+1}\right) / B_{1}\right)\right] \\
& u_{2, k}^{*}=\min \left[u_{2}^{\max }, \max \left(0, K y_{k}\left(\lambda_{3, k+1}-\lambda_{1, k+1}\right) / B_{2}\right)\right]
\end{aligned}
$$

\section{Implementation and interpretation}

In this section, we make use of an iterative method with forward solving of the state system followed by backward solving of the adjoint system and we give the meaning and the estimated value of each parameter in table 5. Also, we present three numerical illustrations using different weight factors. In each example, we achieve relevant results that help us to compare between before and after of these treatments namely the uninfected cells, the infected cells and the viral load.

\subsection{Numerical method and estimated parameters}

In this study, we will implement numerical method for solving this problem. This system consists of the state system, adjoint system, initial and final time conditions, and the controls characterization.

The optimality system is given by (14)

$$
\begin{aligned}
& x_{k+1}=s+x_{k}-\left(1-u_{1, k}^{*}\right) \beta v_{l_{2} k} x_{k}-d x_{k}+r y_{k} \\
& y_{k+1}=y_{k}+\left(1-u_{1, k}^{*}\right) \beta v_{l_{k} k} x_{k}-(a+r) y_{k} \\
& v_{I_{2} k+1}=v_{l_{l, k}}+\left(1-u_{2, k}^{*}\right) K y_{k}-\mu v_{l_{l} k} \\
& v_{N, k+1}=v_{N, k}+u_{2, k}^{*} \rho K y_{k}-\mu v_{N, k} \\
& \lambda_{1, k}=\lambda_{1, k+1}\left[-(d-1)-\left(1-u_{1, k}^{*}\right) \beta v_{l, k}\right] \\
& +\lambda_{2, k+1}\left[\left(1-u_{1, k}^{*}\right) \beta v_{l_{l} k}\right] \\
& \lambda_{2, k}=A+\lambda_{1, k+1} r+\lambda_{2, k+1}[(-a-r-1)] \\
& +\lambda_{3, k+1}\left[\left(1-u_{2, k}^{*}\right) K\right]+\lambda_{4, k+1}\left[u_{2, k}^{*} K\right] \\
& \lambda_{3, k}=C+\left(\lambda_{1, k+1}+\lambda_{2, k+1}\right)\left[-\left(1-u_{1, k}^{*}\right) \beta x_{k}\right] \\
& +\lambda_{3, k+1}[-(\mu-1)] \\
& \lambda_{4, k}=\lambda_{4, k+1}[-(\mu-1)]
\end{aligned}
$$
With these initial conditions $x_{0} \geq 0 y_{0} \geq 0 v_{I_{l} 0} \geq 0$

$$
\lambda_{1, T}=\lambda_{4, T}=0, \lambda_{3, T}=C \text { and } \lambda_{2, T}=A .
$$

\section{Volume 5 Issue 2, February 2016 www.ijsr.net}


International Journal of Science and Research (IJSR)

ISSN (Online): 2319-7064

Index Copernicus Value (2013): 6.14 | Impact Factor (2014): 5.611

Table 5: Meanings and values of parameters

\begin{tabular}{|c|c|c|}
\hline Parameters & Meanings & Values \\
\hline $\mathrm{d}$ & Death rate of uninfected CD4 ${ }^{+}$T-cells population & $0.02 \mathrm{~d}^{-1}$ \\
\hline $\mathrm{a}$ & Death rate of uninfected CD4 $\mathrm{d}^{+}$-cells population & $0.5 \mathrm{~d}^{-1}$ \\
\hline$\mu$ & Death rate of infectious and noninfectious virus & 6 vir.cell $^{-1} \cdot \mathrm{d}^{-1}$ \\
\hline $\mathrm{K}$ & Virion production by infected cells & $0.01 \mathrm{~d}^{-1}$ \\
\hline $\mathrm{r}$ & Rate of cure & $0.000024 \mathrm{~mm}^{3} \cdot \mathrm{vir}^{-1} \cdot \mathrm{d}^{-1}$ \\
\hline$\beta$ & Rate of virion infection of CD4 & $10 \mathrm{cell}^{+} \cdot \mathrm{mm}^{-3} \cdot \mathrm{d}^{-1}$ \\
\hline $\mathrm{s}$ & Source term for uninfected CD4 \\
\hline
\end{tabular}

control has a duration of 75 days, whereas the second control

There are initial conditions for the state variables and terminal conditions for the adjoints. That is, the optimality system is a two-point boundary value problem, with separated boundary conditions at times step $k=0$ and $k=T$. We solve the optimality system by an iterative method with forward solving of the state system followed by backward solving of the adjoint system. We start with an initial guess for the controls at the first iteration and then before the next iteration, we update the controls via the characterization [7]. We exploit several resources [8],[9],[10],[11],[12],[13] in order to extract the different estimated parameters. So, a complete list of parameters and their estimated values are given in table 5 .

Chemotherapy has potentially hazardous side effects. Therefore, the length of treatment is restricted. For most HIV chemotherapy drugs, the length of treatment is less than 500 days. In addition, the optimal controls will depend implicitly on the length of the treatment, and the general shape of the optimal controls stays the same as the treatment interval changes [14]. Hence, the period of the therapy considered at the first is 100 days.

In the following simulations, we adopt initial conditions given by: $x_{0}=1000$ cell. $\mathrm{mm}^{-3}, y_{0}=100$ cell. $\mathrm{mm}^{-3}$,

$v_{I_{2} 0}=30 \mathrm{vir} . \mathrm{mm}^{-3}$ and $v_{N_{2} 0}=0$ vir. $\mathrm{mm}^{-3}$.

\subsection{Numerical Illustration 1}

In the first illustration, the weight factors are given by: $\mathrm{A}=20, \mathrm{~B}_{1}=3000, \mathrm{~B}_{2}=2000$ and $\mathrm{C}=500$. The figure 1 gives us the opportunity to compare easily the behaviors of the two discrete HIV models, before and after therapy with Protease Inhibitors (PIs) and Reverse Transcriptase Inhibitors (RTIs). In addition to this, optimal chemotherapy strategy for the HIV infection is viewed on the figure 2. In fact, Fig1.(a) shows that the concentration of uninfected $\mathrm{CD}_{4}{ }^{+} \mathrm{T}$-cells is higher in case of therapy. Fig1.(b) reveals that in presence of treatment concentration of infected $\mathrm{CD}_{4}{ }^{+}$ $\mathrm{T}$-cells decreases rapidly. In Fig1.(c) we remark that in the absence of therapy infectious virus reach the top at 913 cell. $\mathrm{mm}^{-3}$ in 3 days and decrease rapidly towards zero in the $60^{\text {th }}$ day, whereas in the presence of therapy virus reach just 810 cell. $\mathrm{mm}^{-3}$ in one day and decreases asymptotically to zero in the $20^{\text {th }}$ day. In Fig1.(d) the concentration of noninfectious virus grows rapidly until 402 vir. $\mathrm{mm}^{-3}$ in three days but decreases exponentially until disappearance after 30 days of treatment.

On the whole, we can deduce that introduction of therapy by Protease Inhibitors (PIs) and Reverse Transcriptase Inhibitors (RTIs) has a powerful rule to fight HIV infection and improve the life of the patient. These beneficial results are obtained by the treatment described in Fig.2. The first has duration of 80 days. Hence, the treatment lasts 80 days.

\subsection{Numerical Illustration 2}

In the second illustration, the weight factors are given by: $\mathrm{A}=1000, \mathrm{~B}_{1}=500, \mathrm{~B}_{2}=1000$ and $\mathrm{C}=200$. Evolution of cells and virus is described clearly in the Fig. 3 which permits us to confirm that treatment is able to reduce more and more concentration of infected cells and infectious virus. Despite changing weight factors, the result related to concentration of uninfected cells, infected cells, infectious virus and noninfectious virus differ but slightly, in other words Fig.3 don't bring an extra supply. Unlike optimal controls, adopted in this second simulation, are different to optimal controls in the first simulation, see Fig. 2 and Fig.4, whereas the treatment here has the same duration 80 days.

\subsection{Numerical Illustration 3}

In the last illustration, the weight factors are given by: $\mathrm{A}=3000, \mathrm{~B}_{1}=50, \mathrm{~B}_{2}=10$ and $\mathrm{C}=400$. In this subsection, we vary the weight factors once more, but evolution of uninfected cells, infected cells, infectious virus and noninfectious virus is almost the same compared to the first simulation, see Fig.1 and Fig.5. According to Fig.6, the first control has a duration of 75 days, whereas the second control has duration of 92 days. Consequently, the treatment requires a longer period 92 days.

\section{Conclusion}

This research is part of a multidisciplinary subject and its objective was to study mathematical modeling and optimal control of HIV/AIDS infection. Our approach consists of modeling in discrete time dynamics evolution of the infection, mainly the dynamics of viral load in order to predict its evolution and adopt a medical and economic strategy in a relevant and efficient way.

Despite these many scientific advances due in particular to chemical engineering, genetic engineering and biomedical, HIV/AIDS remains a major scientific problem.

Antiretroviral (ART) that exist allow to lower the viral load in the patient in order to slow the depletion of the immune system but eradication of virus from the body is still impossible. In addition to the high costs of these ART, treatments are very heavy for the patient considering their severe side effects (neurological disorders, acute diarrhea, metabolic disorders, etc.) and often irreversible [8], [15].

To evaluate this work and to establish better treatment strategy, we have investigated three different numerical simulations and have achieved many objectives and results.

\section{Volume 5 Issue 2, February 2016 www.ijsr.net}




\section{International Journal of Science and Research (IJSR) \\ ISSN (Online): 2319-7064}

Index Copernicus Value (2013): 6.14 | Impact Factor (2014): 5.611

First, the treatment period was reduced to 92 days in the worst case. Second, without controls, infected cells and viral load decrease at a slower rate than in case of control with two therapies (PIs) and (RTIs). These latest results confirm the effectiveness of the optimal control in terms of time and in terms of improvement of the health of patients. To end, another point to raise is that despite the change of weight factors in each illustration, the results at the patient's health are beneficial and almost identical, while the treatment varied from one case to another in terms of distribution and duration.

The purpose of this work is to offer intelligent tools that will serve the field of biomedicine and can help the public health authorities to intervene in the right time and with the right way. Not to mention that, according to results approved in this research, governmental organizations, non-governmental organizations and the international community should react as soon as possible to provide ART especially in low and middle income countries to preserve the life of humanity.

\section{References}

[1] W. Garira, S. Musekwa, T. Shiri, Optimal control of combined therapy in a single strain HIV-1 model. Electronic Journal of Differential Equations, 2005, 52: $1-22$.

[2] H. Joshi, Optimal Control of an HIV Immunology Model. Applied Mathematics Optimization 23: 199213, 2002.

[3] D. Kirschner, S. Lenhart, S. Serbin, Optimal control of the chemotherapy of HIV. Journal of Mathematical Biology 35: 775-792, 1997.

[4] W.H. Fleming, R.W. Rishel, Deterministic and stochastic optimal control, Springer, New York, 1975.

[5] D.L. Lukes, Differential equations: classical to controlled, Math Sci Eng 162, Academic Press, New
York, 1982.

[6] L. S Pontryagin, V. G. Boltyanskii, R. V. Gamkrelidze, E.F. Mishchenko, The Mathematical Theory of Optimal Processes, Wiley, New York, 1962.

[7] O. Balatif, M. Elhia, J. Bouyaghroumi and M. Rachik, Optimal Control Strategy for a Discrete SIR Epidemic Model, International Journal of Applied Mathematics and Modeling IJA2M, Vol.2, No. 2, 1-8, 2014.

[8] JF. Delfraissy, Prise en charge thrapeutique des personnes infectes par le VIH: Recommandations du groupe dexperts. Mdecine Science, Paris, flammarion edition, 2002.

[9] M. A. Nowak and R. M. May, Virus dynamics: Mathematical principles of immunology and virology. Oxford University Press, 2002.

[10] David D. Ho et al., Rapid turnover of plasma virion and CD4 lymphocytes in HIV-1 infection. Nature 373: 123126, January 1995.

[11]D. Kirschner and G. F. Webb, A model for treatment strategy in the chemotherapy of AIDS. Bulletin of Mathematical Biology 58:367-390, 1996.

[12]X. Xia and C. H. Moog, Identifiability of nonlinear systems with application to HIV/AIDS models. IEEE Transactions on Automatic Control 48(2):330-336, February 2003.

[13]A. S. Perelson et al., Decay characteristics of HIV-1 infected compartment during combination therapy. Nature, 387:188-191, 1997.

[14] R. Fister, S. Lenhart, J. McNally, Optimizing chemotherapy in an HIV model. Journal of Differential Equations 32: 112, 1998.

[15] H. Banks, T. Jang, H. Kwon, Feedback control of HIV antiviral therapy with long measurement time. International Journal of Pure and Applied Mathematics 66: 461-485, 2011.

\section{Figures List}

(a) Uninfected $\mathrm{CD}_{4}^{+} \mathrm{T}$-cells

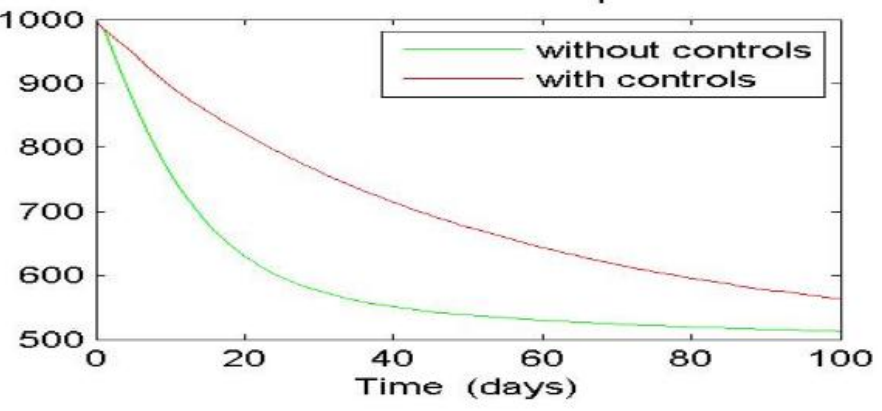

(c) Infectious virus

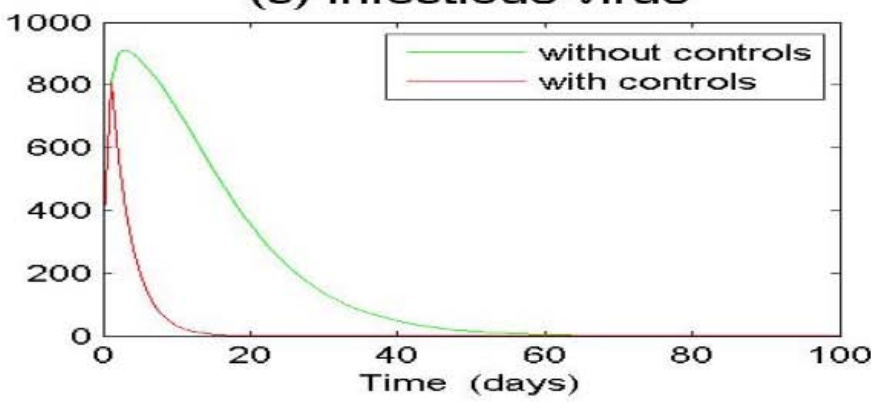

(b) Infected $\mathrm{CD}_{4}^{+} \mathrm{T}$-cells

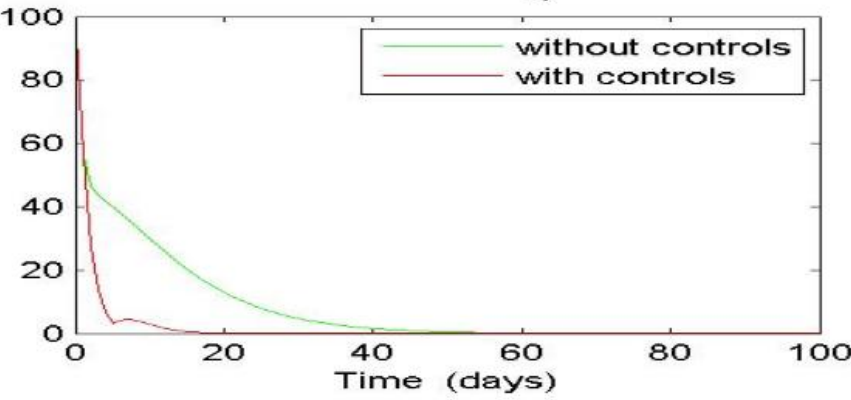

(d) Noninfectious virus

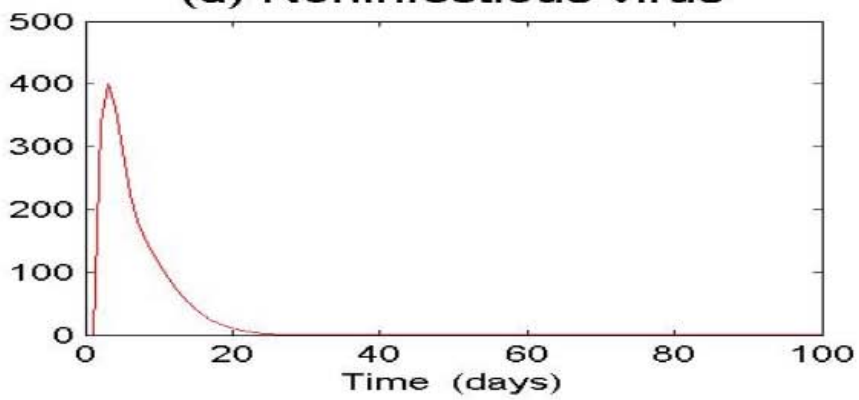

Figure 1: Simulation 1. (a) Evolution of unifected cells in two cases: with controls and without controls. (b) Evolution of

Volume 5 Issue 2, February 2016 www.ijsr.net

Licensed Under Creative Commons Attribution CC BY 


\section{International Journal of Science and Research (IJSR) \\ ISSN (Online): 2319-7064}

Index Copernicus Value (2013): 6.14 | Impact Factor (2014): 5.611

infected cells in two cases: with controls and without controls. (c) Infectious virus in the absence of therapy and during therapy. (d) Noninfectious virus appeared thanks to Protease Inhibitors (PIS)
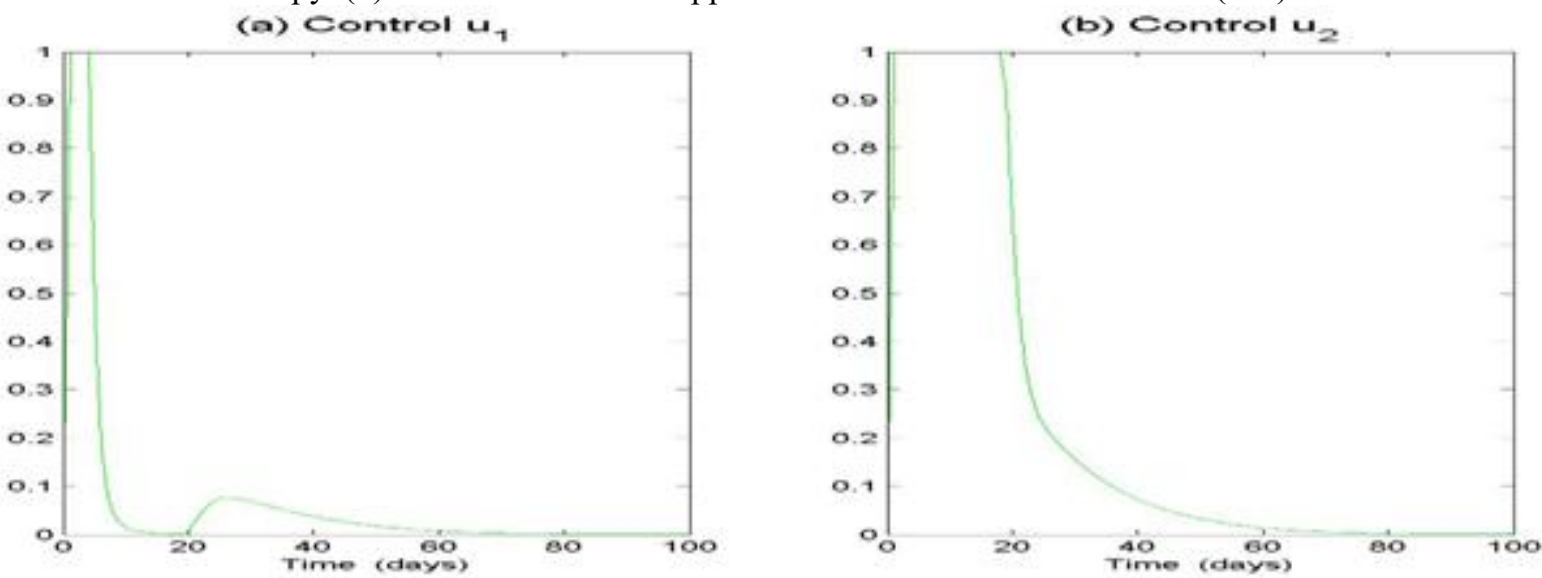

Figure 2: Simulation 1. (a) Optimal control $u_{1}$ (RTIs). (b) Optimal control $u_{2}$ (PIs).

(a) Uninfected $\mathrm{CD}_{4}^{+} \mathrm{T}$-cells

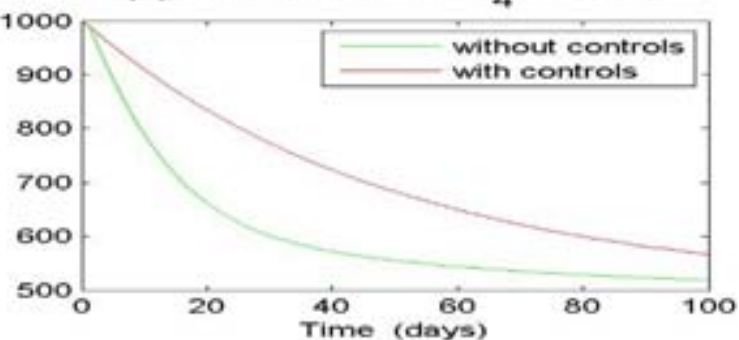

(c) Infectious virus

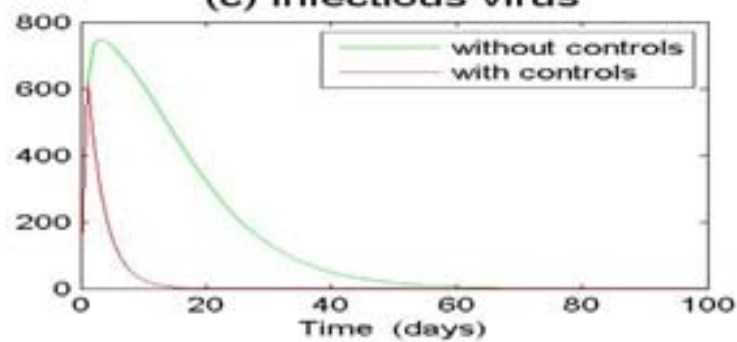

(b) Infected $\mathrm{CD}_{4}^{+} \mathrm{T}$-cells

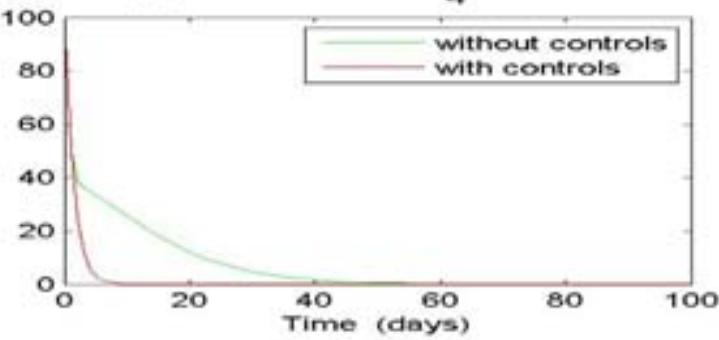

(d) Noninfectious virus

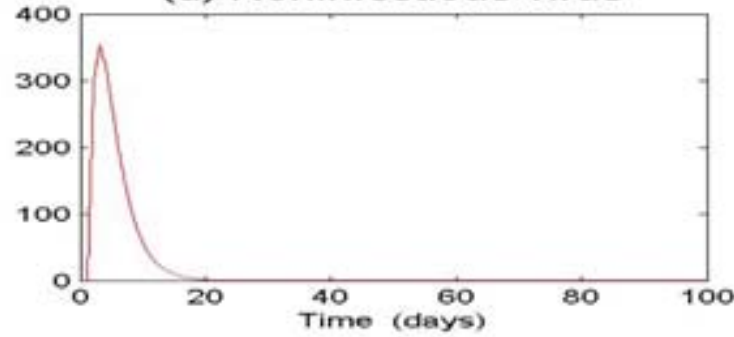

Figure 3: Simulation 2. (a) Evolution of unifected cells in two cases: with controls and without controls. (b) Evolution of infected cells in two cases: with controls and without controls. (c) Infectious virus in the absence of therapy and during therapy. (d) Noninfectious virus appeared thanks to Protease Inhibitors (PIS).
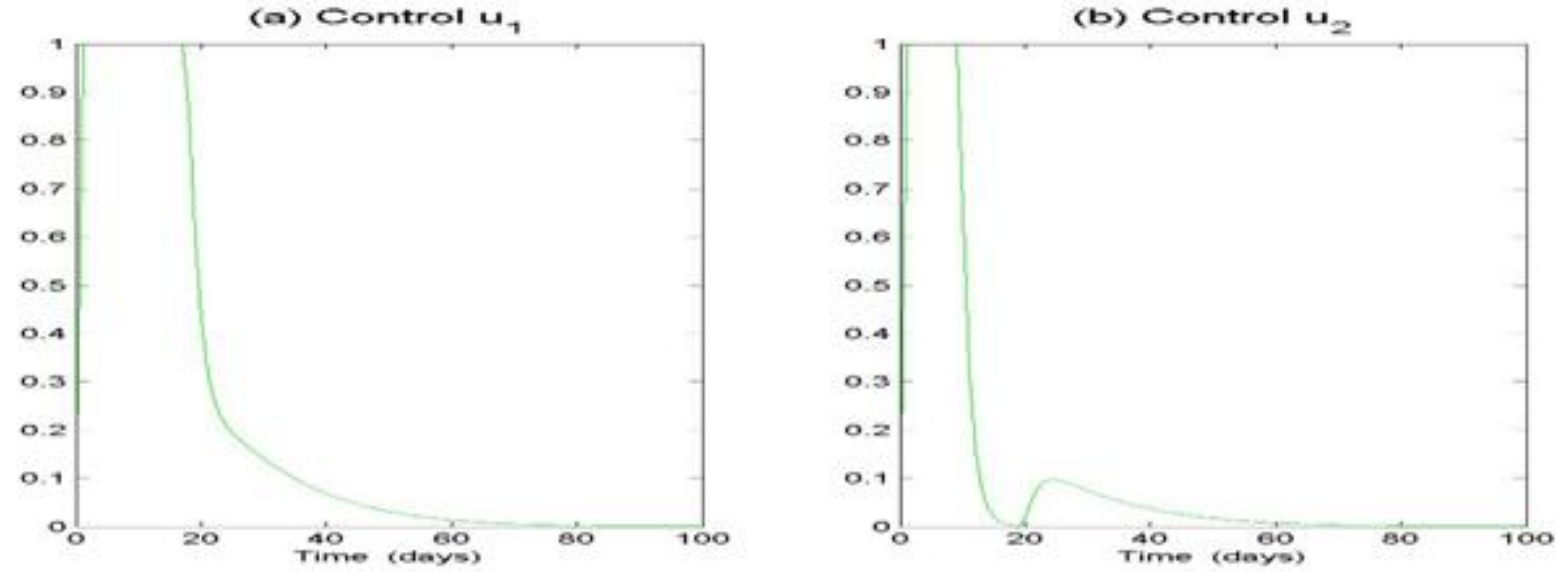

Figure 4: Simulation 2. (a) Optimal control $u_{1}$ (RTIs). (b) Optimal control $u_{2}$ (PIs). 
International Journal of Science and Research (IJSR)

ISSN (Online): 2319-7064

Index Copernicus Value (2013): 6.14 | Impact Factor (2014): 5.611

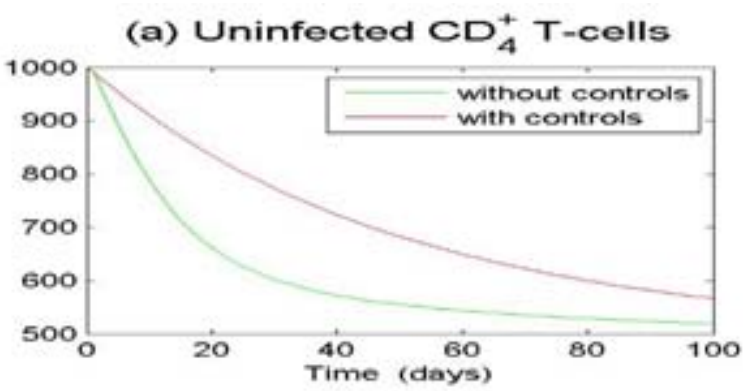

(b) Infected $\mathrm{CD}_{4}^{+} \mathrm{T}$-cells

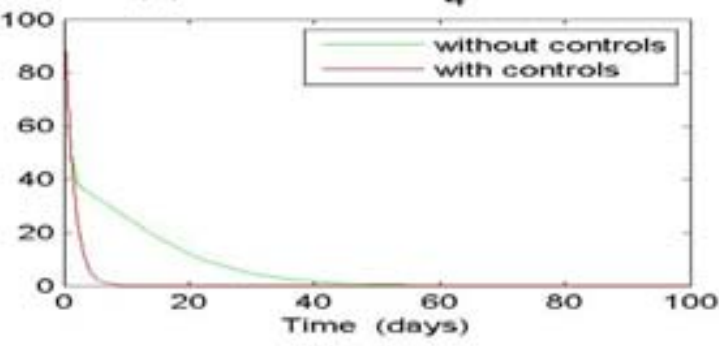

(c) Infectious virus
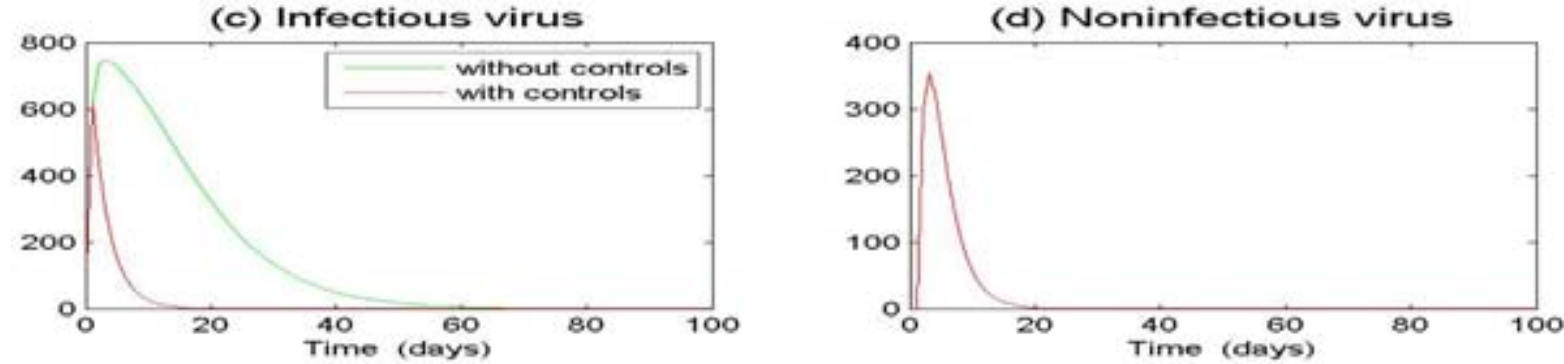

Figure 5: Simulation 3. (a) Evolution of unifected cells in two cases: with controls and without controls. (b) Evolution of infected cells in two cases: with controls and without controls. (c) Infectious virus in the absence of therapy and during therapy. (d) Noninfectious virus appeared thanks to Protease Inhibitors (PIS).
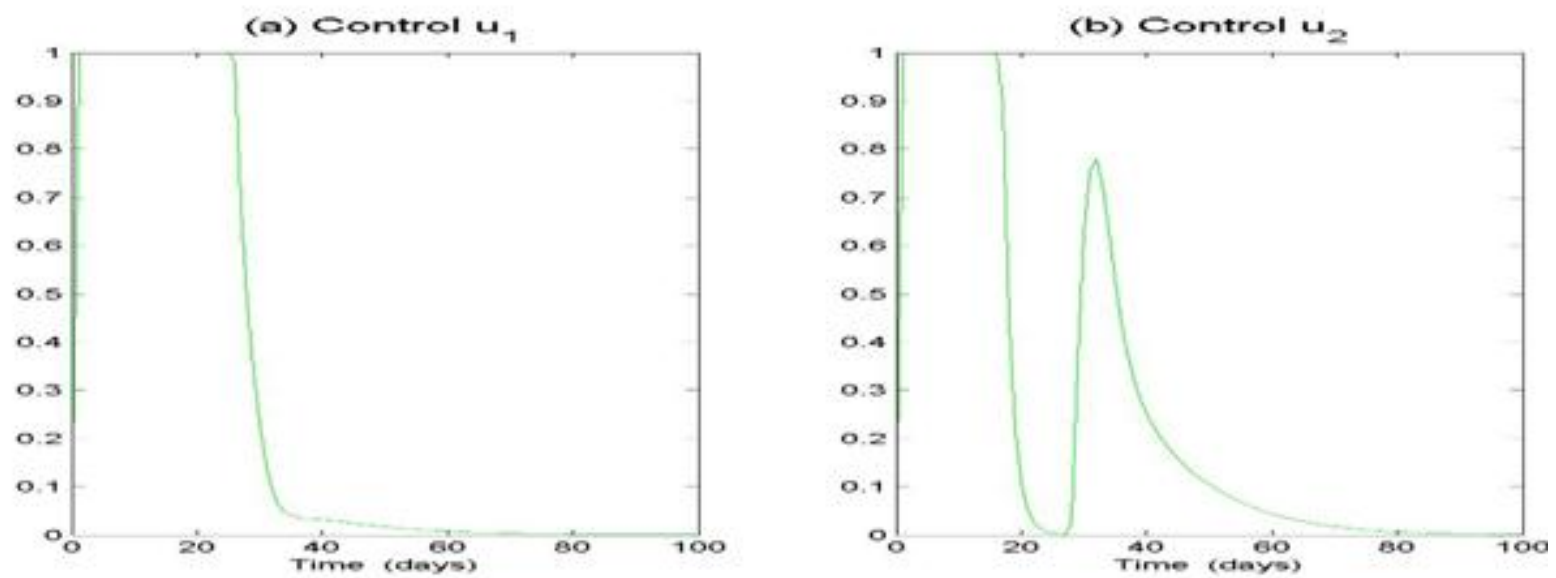

Figure 6: Simulation 3. (a) Optimal control $u_{1}$ (RTIs). (b) Optimal control $u_{2}$ (PIs). 